\title{
Lessons from Multicenter Studies on CSF Biomarkers for Alzheimer's Disease
}

\author{
Niklas Mattsson, Henrik Zetterberg, and kaj Blennow \\ The Clinical Neurochemistry Laboratory, Department of Psychiatry and Neurochemistry, Institute of Neuroscience and Physiology, \\ Sahlgrenska University Hospital, The Sahlgrenska Academy at University of Gothenburg, 43180 Mölndal, Sweden \\ Correspondence should be addressed to Niklas Mattsson, niklas.mattsson@neuro.gu.se \\ Received 19 April 2010; Accepted 10 May 2010 \\ Academic Editor: Lucilla Parnetti \\ Copyright ( 92010 Niklas Mattsson et al. This is an open access article distributed under the Creative Commons Attribution \\ License, which permits unrestricted use, distribution, and reproduction in any medium, provided the original work is properly \\ cited. \\ Several single-center studies have confirmed the usability of cerebrospinal fluid (CSF) biomarkers for the diagnosis of Alzheimer's \\ disease $(\mathrm{AD})$, even in early disease stages. Large scale multicenter studies have principally confirmed this, although such studies \\ have also indicated the presence of significant intercenter and interlaboratory variations in biomarker measurements. Such \\ variations may hamper the development of biomarkers and their introduction into clinical routine practice. Recently a quality \\ control program run by the Alzheimer's Association was started in order to harmonize procedures of laboratories world-wide. \\ This program provides both standardized guide lines and external control CSF samples, and will allow longitudinal evaluation of \\ laboratory performance.
}

\section{Introduction}

The ongoing development of disease modifying treatment for Alzheimer's disease $(\mathrm{AD})$ puts pressure on researchers to develop reliable biomarkers for diagnosis, disease progression and monitoring of treatment effect. For these purposes, the most promising biomarkers are imaging and cerebrospinal fluid (CSF) markers [1-3]. The core CSF biomarkers are $\beta$-amyoid42 (A $\beta 42)$, total-tau (T-tau), and phosphorylated tau (P-tau), where the most frequently used P-tau isoforms are tau phosphorylated at the threonine amino acid residues 181 or 231 [1]. CSF A $\beta 42$, T-tau and Ptau correspond to the principal neuropathological elements of $\mathrm{AD}$ : extracellular amyloid plaques, axonal degeneration, and intraneuronal tangles. Indeed, autopsy studies and imaging studies have revealed that CSF biomarker concentrations correspond well to brain alterations [4-7]. The accessibility of CSF for analysis and the low cost of CSF biomarker measurements favor their usage for detection, and monitoring of pathological processes in the brain. Several studies have shown that AD patients have decreased CSF $\mathrm{A} \beta 42$ and increased T-tau and P-tau compared with healthy controls [8]. Noteworthy, T-tau and P-tau correlate in AD patients and controls but not in several other neurological diseases. T-tau is increased in several neurodegenerative conditions and is a sensitive but unspecific $\mathrm{AD}$ biomarker [9]. P-tau, on the other hand, may add specificity for AD in dementia investigations [10].

\section{CSF Biomarkers in Early Diagnosis}

Brain alterations are likely to start long before onset of clinical dementia. Thus, biomarkers that detect changes in the brain are likely altered at a clinically early stage. It has been proposed that biomarker alterations occur dynamically during the disease process, with $\mathrm{A} \beta$ and Tau markers changed first, followed by distortion of brain structure, decline of memory and ultimately clinical dysfunction [11]. Supporting the use of CSF biomarkers for early diagnosis, decreased $\mathrm{A} \beta 42$ and increased T-tau and P-tau are seen in patients with mild cognitive impairment (MCI) later diagnosed with $\mathrm{AD}$. In a well-controlled monocenter study, Hansson and coworkers followed 137 MCI patients for 4-6 years, during which 57 patients were diagnosed with AD. The CSF biomarkers sampled at baseline had 
a sensitivity of $95 \%$ and a specificity $83 \%$ for incipient $\mathrm{AD}$ [12]. Since clinical AD diagnosis lacks some accuracy versus autopsy confirmation, it is probably not possible to achieve a significantly higher diagnostic accuracy for biomarkers towards clinical diagnosis. In line with this, consensus reports recommend that $\mathrm{AD}$ biomarkers should have at least $85 \%$ sensitivity and $75 \%-85 \%$ specificity [13]. As demonstrated by the study by Hansson and coworkers as well as other studies [14-20], this goal is achievable with the CSF biomarkers A $\beta 42$, T-tau, and P-tau even in early stages.

\section{Multicenter Studies of Diagnostic Accuracy}

The results from the above mentioned studies are encouraging, but it should be noted that diagnostic biomarkers generally perform better in homogenous monocenter studies than in more challenging heterogeneous multicenter studies. So far, only a few large scale multicenter studies of CSF AD biomarkers have been published. In the DESCRIPA study, by Visser and coworkers, CSF samples were taken from 193 MCI and (subjective cognitive impairment) SCI patients and 89 controls at 9 centers in Europe [21]. All samples were analyzed at one laboratory. The results confirmed that a CSF AD profile, as defined by Hulstaert et al. [22], predicted $\mathrm{AD}$ in MCI with an odds ratio of 27 (95\% CI 1.6-460) in amnestic MCI patients. However, the study was complicated by the fact that all controls were enrolled at one center, and CSF sampling procedures differed between centers. Also, $31 \%$ of healthy controls presented a CSF AD pattern, indicating a lack of specificity of the CSF biomarkers. This finding may seem controversial but is consistent with a by now large body of literature, showing that around one third of cognitively normal elderly display AD-like changes in their brains at autopsy [23], in their CSF [24, 25] or on PET scans imaging amyloid [26-28]. Longitudinal studies will tell us whether these individuals do have preclinical AD, or whether silent $\mathrm{AD}$ pathology is part of the normal aging process. Recent studies using repeated cognitive assessments [29] or functional magnetic resonance imaging measures of cortical network integrity [30] favor the first of the two scenarios.

Shaw and coworkers have published CSF biomarker results from the Alzheimer's Disease Neuroimaging Initiative (ADNI) [31]. The ADNI is a multicenter, longitudinal study, launched in 2004. In this study, optimal biomarker cut-offs where established in autopsy confirmed ADNI-independent $\mathrm{AD}$ patients and normal controls, with diagnostic accuracies ranging from $70 \%$ to $87 \%$. The ADNI cohort was recruited at 56 clinical centers, which potentially could introduce large center-dependent variations. To minimize such variations, all participating centers followed the ADNI procedure manual. All samples were analyzed at the ADNI Biomarker Core laboratory at the University of Pennsylvania. The study included $196 \mathrm{MCI}$ patients and 37 of these had been diagnosed with $\mathrm{AD}$ at the 12-month follow-up. A majority $(87-89 \%)$ of these 37 presented a CSF AD profile at baseline. This similar to what was seen in patients with mild $\mathrm{AD}$ at baseline $(\mathrm{N}=100,88 \%-91 \%)$. However, as in the DESCRIPA study, a large proportion (34\%-38\%) of cognitively healthy controls $(\mathrm{N}=114)$ had the same biomarker pattern, again indicating a lack of specificity in relation to clinical diagnosis.

In a third multicenter investigation, published in JAMA in 2009, we enrolled $750 \mathrm{MCI}$ patients, $529 \mathrm{AD}$ patients, and 304 healthy controls from 12 centers in Europe and the United States [32]. Four laboratories were involved, enabling evaluation not only of Intercenter, but also of interlaboratory differences, as discussed below. Cut-offs for the combination of $\mathrm{A} \beta 42, \mathrm{~T}$-tau, and P-tau were constructed in $\mathrm{AD}$ patients and controls, with sensitivity $85 \%$ in accordance with the above mentioned consensus document. This yielded $88 \%$ specificity towards healthy controls. In MCI patients followed for at least 2 years, the sensitivity of the biomarkers was $83 \%$ and the specificity $72 \%$. The lower specificity compared to the monocenter study by Hansson et al. could partly be explained by the shorter followup, which makes it hard to verify the benign nature of all stable MCI cases. However, Intercenter variations may also have influenced the results and blurred some effects of the biomarkers. For the remaining part of this review, we will focus on such variations, and possible ways of eliminating them.

\section{Intercenter Biomarker Variations}

CSF studies on $\mathrm{AD}$ patients and controls report different biomarker concentrations, reference ranges, and diagnostic cut-offs [8]. CSF A $\beta 42$ levels in AD patients in some studies even exceed the levels in controls in other studies. Such fluctuations make it hard or impossible to compare CSF biomarkers between centers and studies. These Intercenter variations come as no surprise. Rather, it is more rule than exception that a novel clinical chemical measurement present variations between centers, due to preanalytical or analytical confounding factors. Such variations are traditionally tackled by quality control programs, which until recently have been lacking for CSF dementia markers. For the CSF biomarkers $\mathrm{A} \beta 42, \mathrm{~T}$-tau, and $\mathrm{P}$-tau, possible confounding factors include preanalytical, analytical, and assay factors [33-35]. Preanalytical factors include, for example, usage of different test tubes and differences in sample handling and storage. Analytical factors include differences in pipetting technique and other laboratory procedures. Finally, important assay factors are batch-to-batch variations and differences in standards and coating of antibodies. A growing number of laboratories are performing CSF analyses for dementia diagnostics. To facilitate the use of biomarkers in research settings and to enable their implementation in clinical routine, the Intercenter variations must be dealt with. Basic CSF parameters, including albumin and immunoglobulin levels, typically have interlaboratory coefficients of variation (CV) below $10 \%$. This is a reasonable ultimate aim also for dementia markers. A handful of multicenter studies have investigated interlaboratory variations in CSF dementia markers, and we will now summarize the results of these studies. 


\section{The First International Quality Control Surveys}

In 2006, Lewczuk and coworkers published the first international quality control survey of neurochemical dementia diagnostics [36]. A ventricular CSF sample was collected from a neurosurgical patient. Fourteen laboratories in Germany, Austria, and Switzerland participated and performed CSF biomarker measurements according to local routines. Different commercially available ELISAs were used for $A \beta$ measurement. Three laboratories used a method from The Genetics Company (Zürich, Switzerland) measuring $\mathrm{A} \beta \mathrm{x}-$ 40 and $\mathrm{A} \beta \mathrm{x}-42$, with no specific $\mathrm{N}$-terminal amino acid capture. Thirteen laboratories used the Innotest (Innogenetics, Ghent, Belgium) ELISA for $\mathrm{A} \beta$, which specifically measures $A \beta 1-42$. Fourteen laboratories used Innotest for T-tau, and 11 laboratories used Innotest for P-tau. Interlaboratory coefficients of variation ranged from $21 \%$ to $38 \%$, with the highest numbers reported for $\mathrm{A} \beta \mathrm{x}-42(38 \%)$ and $\mathrm{A} \beta 1-42(29 \%)$. Most laboratories analyzed samples in duplicates and the intra-assay imprecision was mostly low, exceeding 5\% only for T-tau (5.3\%) and $\mathrm{A} \beta 1-42$ (7.5\%).

Verwey and coworkers published another quality survey in 2009 involving 20 laboratories with measurements performed in 2004 and 2008, allowing evaluation of longitudinal stability [37]. For this study, large CSF pools with different biomarker patterns were constructed and samples were distributed to 13 laboratories in 2004 and to 18 laboratories in 2008. A majority of laboratories used the Innotest ELISA for T-tau and P-tau, but in the 2008 run some laboratories had introduced the Luminex method AlzBio3 (Innogenetics, Ghent, Belgium). ELISA methods generally give higher values for CSF biomarkers than Luminex methods, but the methods have equivalent diagnostic accuracy for $\mathrm{AD}$ $[38,39]$. A $\beta 42$ was measured with the Innotest ELISA, or ELISAs from The Genetics Company, Biosource (Invitrogen, Camarillo, USA) or an in-house assay. Three laboratories used the AlzBio3 method for $\mathrm{A} \beta 42$. Interlaboratory $\mathrm{CVs}$ were high for $\mathrm{A} \beta 42$ ELISAs in both 2004 (31\%) and 2008 (37\%), and somewhat lower when only including laboratories using the Innotest $(30 \%$ and $22 \%$, resp.). CVs were smaller for T-tau (2004: 21\%, 2008: 16\%) and P-tau (2004: 13\%, 2008: 15\%). The AlzBio3 method had CVs ranging from $14 \%-22 \%$, but the low number of laboratories performing this assay makes interpretation difficult. Nine laboratories participated in both rounds of the survey and intra-laboratory CVs for these were $25 \%$ for $\mathrm{A} \beta 42,18 \%$ for T-tau and 7\% for P-tau.

These two studies show large interlaboratory variations for $\mathrm{A} \beta 42$, and smaller but significant variations for T-tau and P-tau. Since these studies used centrally distributed control CSF, several preanalytical confounding factors were eliminated. Remaining possible causes of the variations include local differences in analytical routines, machinery differences and batch-to-batch variations in analytical kits. The latter provides a major challenge for kit vendors, and emphasizes the need to have a robust production of antibodies, standard solutions, and analytical plates.

\section{The Swedish Brain Power Survey}

In the multicenter study mentioned above, published in JAMA in 2009, most centers sent their samples to the Clinical Neurochemistry Laboratory in Mölndal for analysis. However, samples from Amsterdam, Munich, and Kuopio, Finland were analyzed at local laboratories. The study therefore provides information about interlaboratory differences. Subset of samples from Amsterdam, Munich and Kuopio were rerun at the laboratory in Mölndal, and values for A $\beta 42$ and T-tau from all three local laboratories differed more than 2CVs from values measured in Mölndal, using Mölndal CVs for the assays (around 10\%). However, even for centers where samples were run at the laboratory in Mölndal, considerable Intercenter variations were seen. Possible sources of these variations include the preanalytical procedures of subject selection, lumbar puncture, sample handling, and storage. Such variations were seen in particular for $\mathrm{A} \beta 42$, but to a less extent also for T-tau and P-tau.

\section{The Alzheimer's Association Quality Control Program}

The studies outlined above present clear evidence for Intercenter and interlaboratory variations in CSF biomarker measurements. This makes it difficult to compare studies, which may hamper development of CSF biomarkers. With the rapid development of disease modifying treatment the $\mathrm{AD}$ scientific community must not lose momentum in biomarker development. Standardization of collection and handling of samples is vital for this, such as suggested by, for example, the German Competence Net Dementias [40]. Accordingly, it was decided at the International Conference on Alzheimer's Disease (ICAD) in Vienna in 2009 to start an international quality control (QC) program for CSF AD biomarkers. This program is run by the Alzheimer's Association and administrated from the Clinical Neurochemistry Laboratory in Mölndal. The program is open for public, private, research, clinical, and pharmaceutical laboratories. Participating laboratories receive a chart with recommended guidelines for lumbar puncture and sample handling and storage. In line with the ADNI procedures manual, the intention of this chart is to harmonize local routines to eliminate confounding factors responsible for Intercenter variations. In a second part of the program, participating laboratories receive QC CSF samples, constructed in Mölndal. These are analyzed and results are reported to the QC program coordinator. The participating laboratories then receive feedback on their analysis compared to the other laboratories. The first round of the program has just been completed and data are being analyzed. The QC program will continue with multiple rounds each year, enabling the tracking of longitudinal changes in performance.

\section{Conclusions}

Multicenter studies have confirmed the high diagnostic accuracy of CSF biomarkers for $\mathrm{AD}$, even at early stage, 
before onset of dementia. In particular, the high diagnostic sensitivity of CSF biomarkers achieved in the ADNI trial shows that harmonization of sample collection and handling allows the usage of the biomarkers even in a widespread multicenter setting. This advocates the use of CSF biomarkers in clinical studies, where they may be used to enrich trials with MCI patients with incipient AD. However, to facilitate the development of biomarkers and to enable their introduction in clinical routine, interlaboratory and Intercenter differences should be systematically analyzed. This is achievable within the Alzheimer's Association QC program.

\section{Disclosures}

Kaj Blennow has served in a scientific advisory board for Innogenetics. Henrik Zetterberg has served in a scientific advisory board for GlaxoSmithKline.

\section{References}

[1] K. Blennow, H. Hampel, M. Weiner, and H. Zetterberg, "Cerebrospinal fluid and plasma biomarkers in Alzheimer disease," Nature Reviews Neurology, vol. 6, no. 3, pp. 131-144, 2010.

[2] G. B. Frisoni, et al., "The clinical use of structural MRI in Alzheimer disease," Nature Reviews Neurology, vol. 6, no. 2, pp. 67-77, 2010.

[3] A. Nordberg, J. O. Rinne, A. Kadir, and B. Lngström, “The use of PET in Alzheimer disease," Nature Reviews Neurology, vol. 6, no. 2, pp. 78-87, 2010.

[4] D. Strozyk, K. Blennow, L. R. White, and L. J. Launer, "CSF $A ß 42$ levels correlate with amyloid-neuropathology in a population-based autopsy study," Neurology, vol. 60, no. 4, pp. 652-656, 2003.

[5] T. Tapiola, I. Alafuzoff, S.-K. Herukka et al., "Cerebrospinal fluid $\beta$-amyloid 42 and tau proteins as biomarkers of Alzheimer-type pathologic changes in the brain," Archives of Neurology, vol. 66, no. 3, pp. 382-389, 2009.

[6] A. M. Fagan, M. A. Mintun, R. H. Mach et al., "Inverse relation between in vivo amyloid imaging load and cerebrospinal fluid A $\beta 42$ in humans," Annals of Neurology, vol. 59, no. 3, pp. 512$519,2006$.

[7] A. Forsberg, H. Engler, O. Almkvist et al., "PET imaging of amyloid deposition in patients with mild cognitive impairment," Neurobiology of Aging, vol. 29, no. 10, pp. 1456-1465, 2008.

[8] K. Blennow and H. Hampel, "CSF markers for incipient Alzheimer's disease," Lancet Neurology, vol. 2, no. 10, pp. 605613, 2003.

[9] N. Mattsson, K. Blennow, and H. Zetterberg, "CSF biomarkers: pinpointing alzheimer pathogenesis," Annals of the New York Academy of Sciences, vol. 1180, pp. 28-35, 2009.

[10] H. Hampel, K. Blennow, L. M. Shaw, Y. C. Hoessler, H. Zetterberg, and J. Q. Trojanowski, "Total and phosphorylated tau protein as biological markers of Alzheimer's disease," Experimental Gerontology, vol. 45, no. 1, pp. 30-40, 2010.

[11] C. R. Jack Jr., D. S. Knopman, W. J. Jagust et al., "Hypothetical model of dynamic biomarkers of the Alzheimer's pathological cascade," The Lancet Neurology, vol. 9, no. 1, pp. 119-128, 2010.
[12] O. Hansson, H. Zetterberg, P. Buchhave, E. Londos, K. Blennow, and L. Minthon, "Association between CSF biomarkers and incipient Alzheimer's disease in patients with mild cognitive impairment: a follow-up study," Lancet Neurology, vol. 5, no. 3, pp. 228-234, 2006.

[13] "Consensus report of the Working Group on: "molecular and biochemical markers of Alzheimer's disease". The Ronald and Nancy Reagan Research Institute of the Alzheimer's Association and the National Institute on Aging Working Group," Neurobiology of Aging, vol. 19, no. 2, pp. 109-116, 1998.

[14] M. Riemenschneider, N. Lautenschlager, S. Wagenpfeil, J. Diehl, A. Drzezga, and A. Kurz, "Cerebrospinal fluid tau and $\beta$-amyloid 42 proteins identify Alzheimer disease in subjects with mild cognitive impairment," Archives of Neurology, vol. 59, no. 11, pp. 1729-1734, 2002.

[15] H. Zetterberg, L.-O. Wahlund, and K. Blennow, "Cerebrospinal fluid markers for prediction of Alzheimer's disease," Neuroscience Letters, vol. 352, no. 1, pp. 67-69, 2003.

[16] N. Andreasen, E. Vanmechelen, H. Vanderstichele, P. Davidsson, and K. Blennow, "Cerebrospinal fluid levels of total-tau, phospho-tau and A $\beta 42$ predicts development of Alzheimer's disease in patients with mild cognitive impairment," Acta Neurologica Scandinavica, Supplement, vol. 179, pp. 47-51, 2003.

[17] H. Hampel, S. J. Teipel, T. Fuchsberger et al., "Value of CSF $\beta$-amyloid1-42 and tau as predictors of Alzheimer's disease in patients with mild cognitive impairment," Molecular Psychiatry, vol. 9, no. 7, pp. 705-710, 2004.

[18] S.-K. Herukka, M. Hallikainen, H. Soininen, and T. Pirttilä, "CSF A $\beta 42$ and tau or phosphorylated tau and prediction of progressive mild cognitive impairment," Neurology, vol. 64, no. 7, pp. 1294-1297, 2005.

[19] S.-K. Herukka, S. Helisalmi, M. Hallikainen, S. Tervo, H. Soininen, and T. Pirttilä, "CSF A $\beta 42$, Tau and phosphorylated Tau, APOE $\varepsilon 4$ allele and MCI type in progressive MCI," Neurobiology of Aging, vol. 28, no. 4, pp. 507-514, 2007.

[20] N. Andreasen, L. Minthon, E. Vanmechelen et al., "Cerebrospinal fluid tau and $\mathrm{A} \beta 42$ as predictors of development of Alzheimer's disease in patients with mild cognitive impairment," Neuroscience Letters, vol. 273, no. 1, pp. 5-8, 1999.

[21] P. J. Visser, F. Verhey, D. L. Knol et al., "Prevalence and prognostic value of CSF markers of Alzheimer's disease pathology in patients with subjective cognitive impairment or mild cognitive impairment in the DESCRIPA study: a prospective cohort study," The Lancet Neurology, vol. 8, no. 7, pp. 619-627, 2009.

[22] F. Hulstaert, K. Blennow, A. Ivanoiu et al., "Improved discrimination of $\mathrm{AD}$ patients using $\beta$-amyloid((1-42)) and tau levels in CSF," Neurology, vol. 52, no. 8, pp. 1555-1562, 1999.

[23] G. M. Savva, S. B. Wharton, P. G. Ince, G. Forster, F. E. Matthews, and C. Brayne, "Age, neuropathology, and dementia," New England Journal of Medicine, vol. 360, no. 22, pp. 2302-2309, 2009.

[24] G. Li, I. Sokal, J. F. Quinn et al., "CSF tau/A $\beta 42$ ratio for increased risk of mild cognitive impairment: a follow-up study," Neurology, vol. 69, no. 7, pp. 631-639, 2007.

[25] E. Stomrud, O. Hansson, K. Blennow, L. Minthon, and E. Londos, "Cerebrospinal fluid biomarkers predict decline in subjective cognitive function over 3 years in healthy elderly," Dementia and Geriatric Cognitive Disorders, vol. 24, no. 2, pp. 118-124, 2007. 
[26] K. E. Pike, G. Savage, V. L. Villemagne et al., " $\beta$-amyloid imaging and memory in non-demented individuals: evidence for preclinical Alzheimer's disease," Brain, vol. 130, no. 11, pp. 2837-2844, 2007.

[27] M. A. Mintun, G. N. Larossa, Y. I. Sheline et al., “[11C]PIB in a nondemented population: potential antecedent marker of Alzheimer disease," Neurology, vol. 67, no. 3, pp. 446-452, 2006.

[28] C. A. Wiley, B. J. Lopresti, S. Venneti et al., "Carbon 11-labeled Pittsburgh compound B and carbon 11-labeled (R)-PK11195 positron emission tomographic imaging in Alzheimer disease," Archives of Neurology, vol. 66, no. 1, pp. 60-67, 2009.

[29] E. Stomrud, O. Hansson, H. Zetterberg, K. Blennow, L. Minthon, and E. Londos, "Correlation of longitudinal cerebrospinal fluid biomarkers with cognitive decline in healthy older adults," Archives of Neurology, vol. 67, no. 2, pp. 217-223, 2010.

[30] T. Hedden, K. R. A. Van Dijk, J. A. Becker et al., "Disruption of functional connectivity in clinically normal older adults harboring amyloid burden," Journal of Neuroscience, vol. 29, no. 40, pp. 12686-12694, 2009.

[31] L. M. Shaw, H. Vanderstichele, M. Knapik-Czajka et al., "Cerebrospinal fluid biomarker signature in alzheimer's disease neuroimaging initiative subjects," Annals of Neurology, vol. 65, no. 4, pp. 403-413, 2009.

[32] N. Mattsson, H. Zetterberg, O. Hansson et al., "CSF biomarkers and incipient Alzheimer disease in patients with mild cognitive impairment," Journal of the American Medical Association, vol. 302, no. 4, pp. 385-393, 2009.

[33] N. Mattsson, K. Blennow, and H. Zetterberg, "Inter-laboratory variation in cerebrospinal fluid biomarkers for Alzheimer's disease: united we stand, divided we fall," Clinical Chemistry and Laboratory Medicine, vol. 48, no. 5, pp. 603-607, 2010.

[34] P. Lewczuk, G. Beck, H. Esselmann et al., "Effect of sample collection tubes on cerebrospinal fluid concentrations of tau proteins and amyloid $\beta$ peptides," Clinical Chemistry, vol. 52, no. 2, pp. 332-334, 2006.

[35] N. S. M. Schoonenboom, C. Mulder, H. Vanderstichele et al., "Effects of processing and storage conditions on amyloid $\beta$ (142) and tau concentrations in cerebrospinal fluid: implications for use in clinical practice," Clinical Chemistry, vol. 51, no. 1, pp. 189-195, 2005.

[36] P. Lewczuk, G. Beck, O. Ganslandt et al., "International quality control survey of neurochemical dementia diagnostics," Neuroscience Letters, vol. 409, no. 1, pp. 1-4, 2006.

[37] N. A. Verwey, W. M. Van Der Flier, K. Blennow et al., "A worldwide multicentre comparison of assays for cerebrospinal fluid biomarkers in Alzheimer's disease," Annals of Clinical Biochemistry, vol. 46, no. 3, pp. 235-240, 2009.

[38] A. Olsson, H. Vanderstichele, N. Andreasen et al., "Simultaneous measurement of $\beta$-amyloid(1-42), total Tau, and phosphorylated Tau (Thr181) in cerebrospinal fluid by the xMAP technology," Clinical Chemistry, vol. 51, no. 2, pp. 336$345,2005$.

[39] T. S. M. Reijn, M. O. Rikkert, W. J. A. Van Geel, D. De Jong, and M. M. Verbeek, "Diagnostic accuracy of ELISA and xMAP technology for analysis of amyloid $\beta 42$ and tau proteins," Clinical Chemistry, vol. 53, no. 5, pp. 859-865, 2007.

[40] P. Lewczuk, J. Kornhuber, and J. Wiltfang, "The German Competence Net Dementias: standard operating procedures for the neurochemical dementia diagnostics," Journal of Neural Transmission, vol. 113, no. 8, pp. 1075-1080, 2006. 


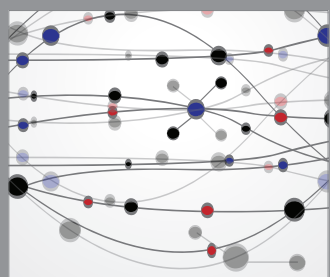

The Scientific World Journal
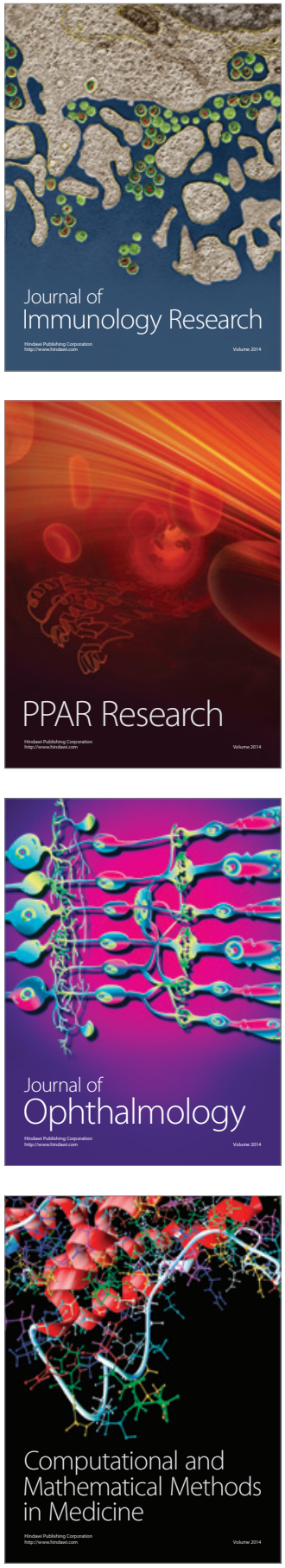

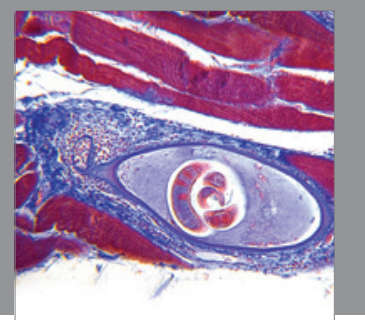

Gastroenterology

Research and Practice
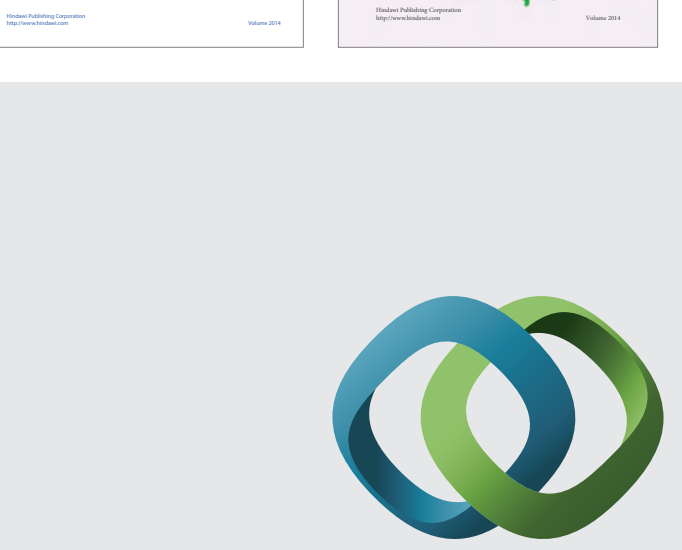

\section{Hindawi}

Submit your manuscripts at

http://www.hindawi.com
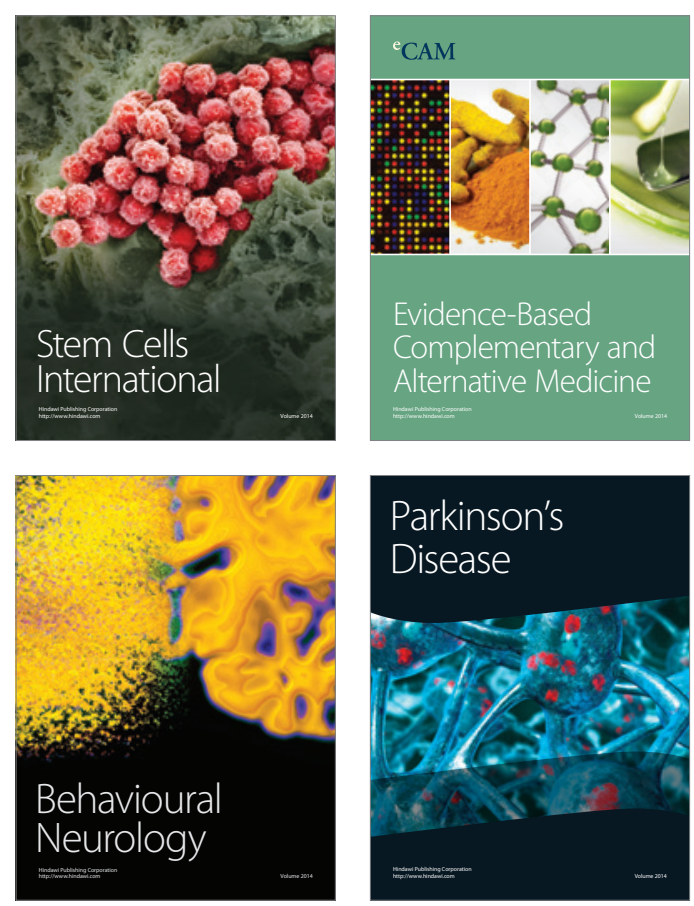

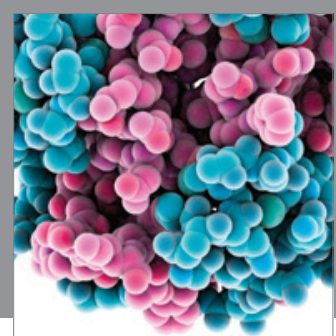

Journal of
Diabetes Research

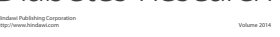

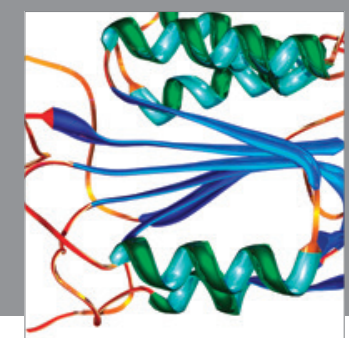

Disease Markers
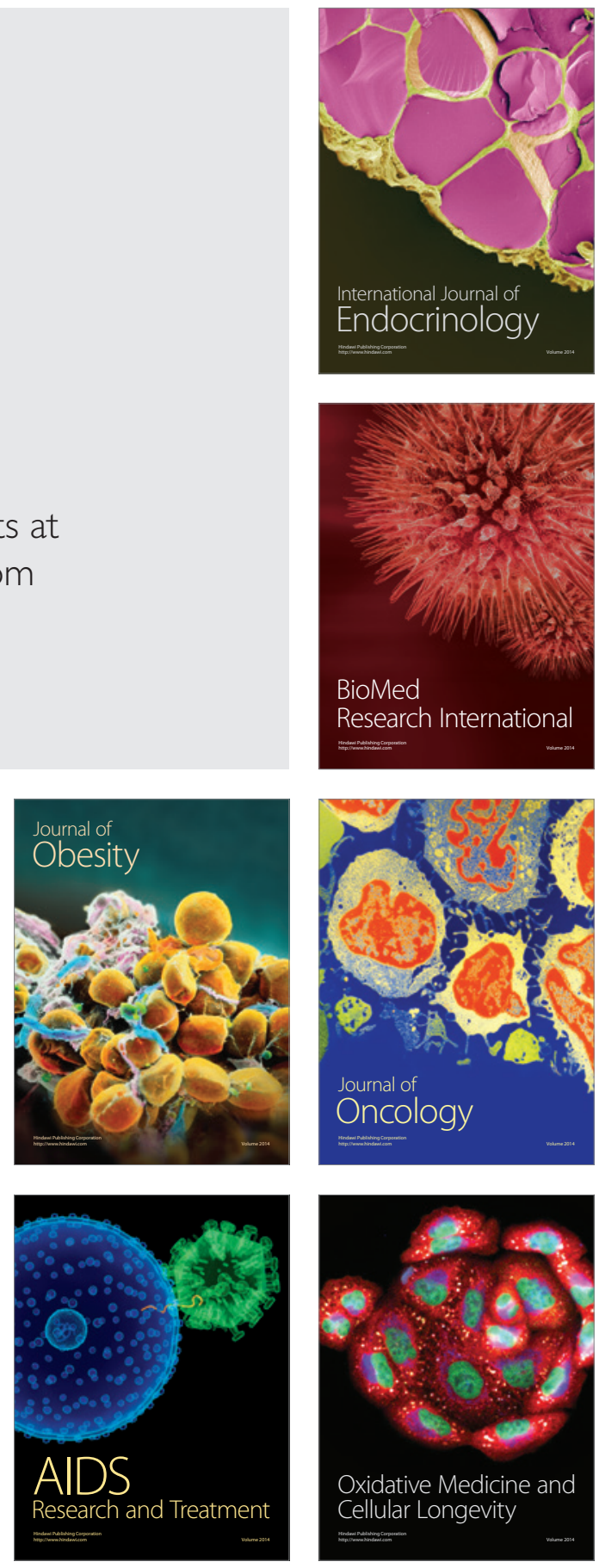